\title{
Profiling of magnesium organosulfur chemistry in meteorites
}

\author{
MARCO MATZKA ${ }^{1}$, MARIANNA LUCIO ${ }^{2}$, BASEM \\ KANAWATI $^{2}$, ERIC QUIRICO ${ }^{3}$, LYDIE BONAL ${ }^{3}$, STEFAN \\ LOEHLE $^{4}$ AND PHILIPPE SCHMITT-KOPPLIN ${ }^{2,5}$ \\ ${ }^{1}$ Helmholtz Zentrum München - Deutsches Forschungszentrum \\ für Gesundheit und Umwelt $(\mathrm{GmbH})$ Finanzabteilung \\ ${ }^{2}$ Helmholtz Zentrum München \\ ${ }^{3}$ Institut de Planétologie et d'Astrophysique de Grenoble \\ ${ }^{4}$ University of Stuttgart \\ ${ }^{5}$ TUM, WZW, Institute of Analytical Food Chemistry \\ Presenting Author: marco.matzka@helmholtz-muenchen.de
}

Organic matter of high molecular diversity is found in terrestrial and extraterrestrial geological samples and invites conclusions about extreme environments such as the Hadean Earth and celestial bodies within the early solar system. Meteorites not only consist of inorganic minerals and metal phases, but also embody an immense diversity of carbon-based molecules. The soluble organic matter of a diverse set of 44 meteorites was analyzed with Fourier transform ion cyclotron resonance mass spectrometry (FT-ICR-MS). The data revealed a distinction between hydrothermal background meteorites (i) without short duration heating, (ii) moderate or (iii) intense short duration heating, or (iv) long duration heating. Although the identification of thermally altered meteorites based on $\mathrm{CHOMg}$ compounds has already been shown [1], the here presented discrimination is solely established on the compositional space of CHOSMg compounds $\left(\mathrm{C}_{\mathrm{x}} \mathrm{H}_{\mathrm{y}} \mathrm{Mg}_{1-2} \mathrm{O}_{\mathrm{z}} \mathrm{S}_{\mathrm{i}}\right.$ with $\left.\mathrm{x}, \mathrm{y}, \mathrm{z}, \mathrm{i} \in \mathbb{N}\right)$ [2]. $\mathrm{CHOSMg}$ compounds in these four groups especially differ in amount of oxygen and sulfur per molecule as well as in saturation. Furthermore, the presented data allows the prediction of unknown samples to categorize them as one of the four groups, which was successfully tested.

Following these lines, we would find CHOSMg compounds in higher abundance in heated meteorites and in their respective asteroid parent bodies. Their properties as organic molecules to survive tremendous heat incidents underline their contribution to our understanding of carbon speciation in harsh extraterrestrial environments. Together with $\mathrm{CHOMg}$ and maybe other metal containing organic compounds, they constitute a heat resistant and durable form of organic compounds in meteorites. Extraterrestrial organic carbon in the form of $\mathrm{CHOSMg}$ compounds benefits from the thermostable characteristics to survive in outer space and travel unscathed for long distances in meteorites as carriers and might be ultimately contributors to the emergence of life.

[1] A. Ruf et al., Previously unknown class of metalorganic compounds revealed in meteorites. Proc Natl Acad Sci U S A 114, 2819-2824 (2017).

[2] M.Matzka et al., Novel magnesium organosulfur chemistry reflects thermal stress in meteorites and asteroid parent bodies. (2021) - Submitted Paper 